\title{
Micromechanics of soil responses in cyclic simple shear tests
}

\author{
Liang Cui ${ }^{1, *}$, Subhamoy Bhattacharya ${ }^{1}$ and George Nikitas ${ }^{1}$ \\ ${ }^{1}$ Department of Civil and Environmental Engineering, University of Surrey, Guildford, GU2 7XH, United Kingdom
}

\begin{abstract}
Offshore wind turbine (OWT) foundations are subjected to a combination of cyclic and dynamic loading arising from wind, wave, rotor and blade shadowing. Under cyclic loading, most soils change their characteristics including stiffness, which may cause the system natural frequency to approach the loading frequency and lead to unplanned resonance and system damage or even collapse. To investigate such changes and the underlying micromechanics, a series of cyclic simple shear tests were performed on the RedHill 110 sand with different shear strain amplitudes, vertical stresses and initial relative densities of soil. The test results showed that: (a) Vertical accumulated strain is proportional to the shear strain amplitude but inversely proportional to relative density of soil; (b) Shear modulus increases rapidly in the initial loading cycles and then the rate of increase diminishes and the shear modulus remains below an asymptote; (c) Shear modulus increases with increasing vertical stress and relative density, but decreasing with increasing strain amplitude. Coupled DEM simulations were performed using PFC2D to analyse the micromechanics underlying the cyclic behaviour of soils. Micromechanical parameters (e.g. fabric tensor, coordination number) were examined to explore the reasons for the various cyclic responses to different shear strain amplitudes or vertical stresses. Both coordination number and magnitude of fabric anisotropy contribute to the increasing shear modulus.
\end{abstract}

\section{Introduction}

Designing foundations for Offshore wind turbine (OWTs) are challenging as these are dynamically sensitive structures in the sense that natural frequencies of these structures are very close to the forcing frequencies [1]. A designer apart from predicting the global natural frequency of the structure, must also ensure that the overall natural frequency due to dynamic-soil-structureinteraction does not shift towards the forcing frequencies $[2,3]$. The global natural frequency is dependent on the soil-structure stiffness, thus an investigation of soil stiffness under cyclic loading is desired. Majority of current OWTs are supported by monopile foundation, which is a large steel tube typically $30-40 \mathrm{~m}$ in length and $3.5-6 \mathrm{~m}$ in diameter. Unlike the slender piles for offshore structure, monopile tends to rotate rather than bend under lateral load or overturning moment. Therefore, the interactions between the monopile and the surrounding soil could be represented by cyclic simple shear tests. Cyclic responses of soil stiffness have been studied intensively $[4,5]$, but few of them were focused on the micromechanism underlying these responses.

The aim of this paper is to study the soil stiffness responses under cyclic simple shear loading with a focus on the micromechanics. Experimental cyclic simple shear tests on typical silica sand (RedHill 100) is first presented. DEM simulations performed using PFC2D are then described, and the macroscopic stiffness and volumetric responses are validated against experiments. The micromechanical parameters are then analysed to find the relationships between the micromechanical parameters and macroscopic responses.

\section{Experimental tests}

Cyclic simple shear apparatus was used for testing cylindrical samples of $50 \mathrm{~mm}$ in diameter and $20 \mathrm{~mm}$ in height. The sample height-to-diameter ratio is 0.4 , agreeing with the ASTM D6528 [6] requirements. The tested soil is RedHill 110 Sand with $\mathrm{d}_{50}=0.18 \mathrm{~mm}$ (PSD curve shown in Fig. 1), a specific gravity $\mathrm{G}_{\mathrm{s}}=2.65$ and minimum and maximum void ratio of 0.608 and 1.035 respectively. Cyclic simple shear tests with different vertical stress $(\sigma)$, relative density $\left(D_{r}\right)$, and shear strain amplitude $\left(\gamma_{\max }\right)$ were performed.

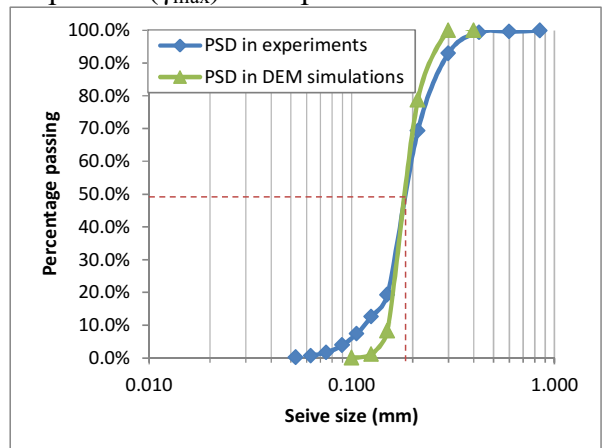

Fig. 1. Particle size distribution (PSD).

The variation of shear modulus and accumulated vertical strain are illustrated in Figs. 2 and 3,

\footnotetext{
Corresponding author: 1.cui@surrey.ac.uk
} 
respectively. The shear modulus increases rapidly in the initial loading cycles and then the rate of increase diminishes and the shear modulus remains below an asymptote. The increase of shear modulus is underlain by the consistent contractive responses of all samples. The shear modulus increases with increasing vertical stress and relative density, but decreases with increasing strain amplitude as expected. It can also be observed that vertical accumulated strain is proportional to the shear strain amplitude but inversely proportional to the relative density of soil. The results correlated quite well with the observations from scaled model tests with different types of offshore wind turbine foundations [2, 3, 7]. The only abnormal trend observed is that the accumulated vertical strain decreases with increasing vertical stress. This is mainly due to the fact that more soil consolidation was resulted by the application of higher vertical stress and the initial relative density has increased to a value much higher than $50 \%$. It could be deduced that the initial relative density has more significant impact on the vertical strain than the vertical stress does. It also shows the difficulties in controlling the relative density for various vertical stresses in sample preparation.

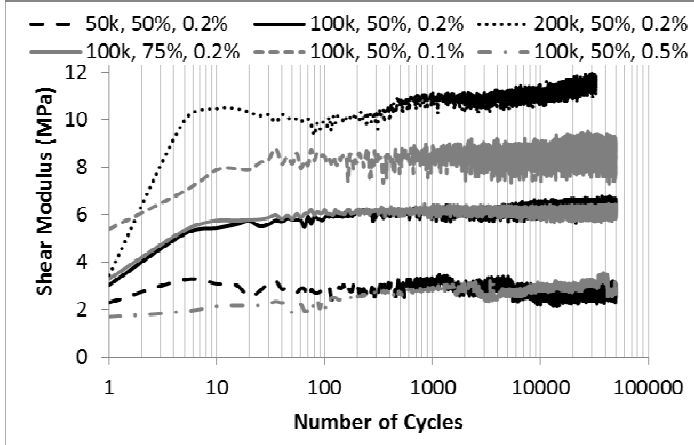

Fig. 2. Variation of shear modulus in experiments

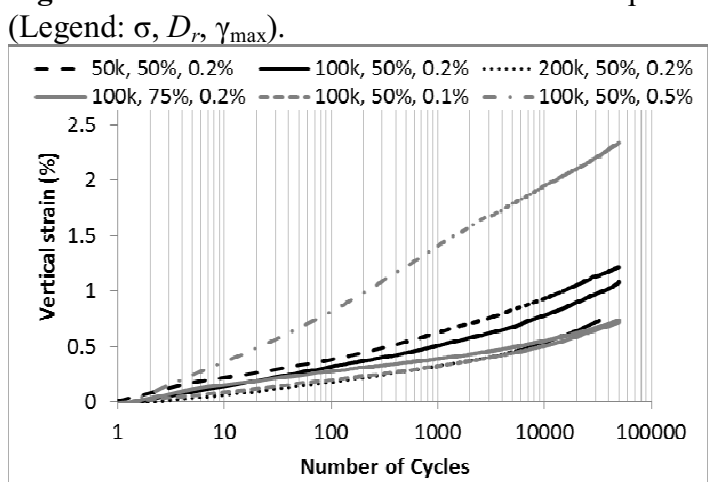

Fig. 3. Accumulated vertical strain in experiments (Legend: $\sigma, D_{r}, \gamma_{\max }$ ).

\section{DEM simulations}

\subsection{Description of DEM setup}

DEM simulations of cyclic simple shear tests were performed using PFC2D [8]. The sample generated for testing is about $20 \mathrm{~mm}$ in height and $50 \mathrm{~mm}$ in width, containing 8000 disks with size ranging from $0.1 \mathrm{~mm}$ to
$0.3 \mathrm{~mm}$ and $\mathrm{d}_{50}=0.18 \mathrm{~mm}$, matching the value in experiments. The PSD curve is also given in Fig. 1. Particle density is $2650 \mathrm{~kg} / \mathrm{m}^{3}$. Inter-particle and particle-boundary frictional coefficient, $\mu$, is 0.5 . Normal stiffness of particle is $8.0 \times 10^{7} \mathrm{~N} / \mathrm{m}$ and shear stiffness of particle is $4.0 \times 10^{7} \mathrm{~N} / \mathrm{m}$. Normal and shear stiffness of boundary are $4.0 \times 10^{9} \mathrm{~N} / \mathrm{m}$. Two sets of samples were generated with radius expansion approach followed by $\mathrm{K}_{0}$ consolidation with $\mu=0$ and 1.0 to generate dense and loose samples, respectively. The void ratios of dense samples at consolidation pressure of $50 \mathrm{kPa}$ and $100 \mathrm{kPa}$ are 0.185 and 0.181 , respectively. The void ratios of loose samples at consolidation pressure of $50 \mathrm{kPa}$ and $100 \mathrm{kPa}$ are 0.215 and 0.227 , respectively. Note that due to different boundary velocities in the consolidation stage, the loose sample reached slightly lower void ratio at $50 \mathrm{kPa}$ than that at $100 \mathrm{kPa}$. 6000 cycles of shear strain in the range of $(0,0.5 \%)$ were applied to each sample. 12 measurement circles were defined within the sample to measure the stress, void ratio and coordination number.

\subsection{Macro-scale responses}

The shear modulus $(G)$ was calculated as the ratio of the difference between shear stress at maximum shear strain $\left(\tau_{\max }\right)$ and shear stress at zero strain $\left(\tau_{0}\right)$ to the maximum shear strain $\left(\gamma_{\max }\right)$. The variations of shear modulus and vertical strain of the four samples under cyclic loading are illustrated in Fig. 4 and Fig. 5, respectively. The magnitudes of shear moduli in the DEM simulations are in the same range as the values in the experimental tests. For both loose samples, there is a clear increase in the shear modulus under cyclic load. For the dense sample with vertical stress $\sigma=100 \mathrm{kPa}$, shear modulus also increases slightly; however, for the dense sample with vertical stress $\sigma=50 \mathrm{kPa}$, shear modulus decreases. After 1000 cycles, the shear moduli of the two loose samples exceed the modulus of one dense sample at low confining pressure.

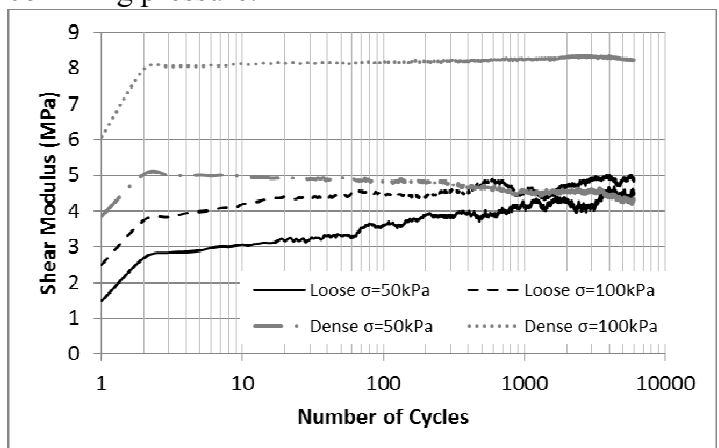

Fig. 4. Evolution of shear modulus in simulations.

The increasing shear modulus could be explained by the densification of loose samples as observed in Fig. 6, which illustrates the evolutions of void ratios in these four samples. However, the different variation trends of stiffness for the two dense samples could not relate directly to the slight dilations of both samples. Moreover, the void ratios of the two loose samples were still much higher than the two dense samples at the end of 6000 loading cycles, but their shear moduli have 
exceeded the value for the dense sample with $\sigma=50 \mathrm{kPa}$. These observations should be explored in more details by considering their particle-scale parameters.

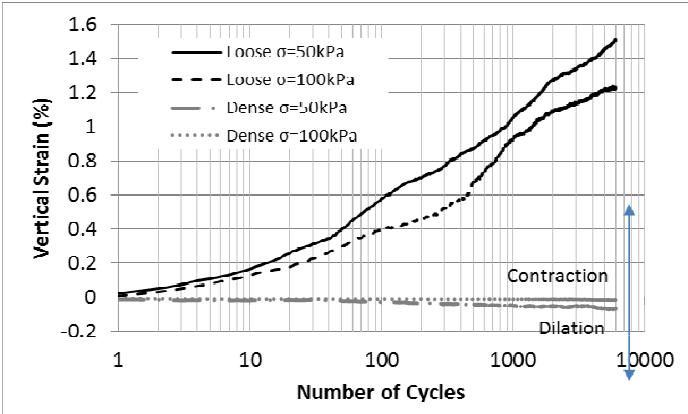

Fig. 5. Accumulated vertical strain in simulations.

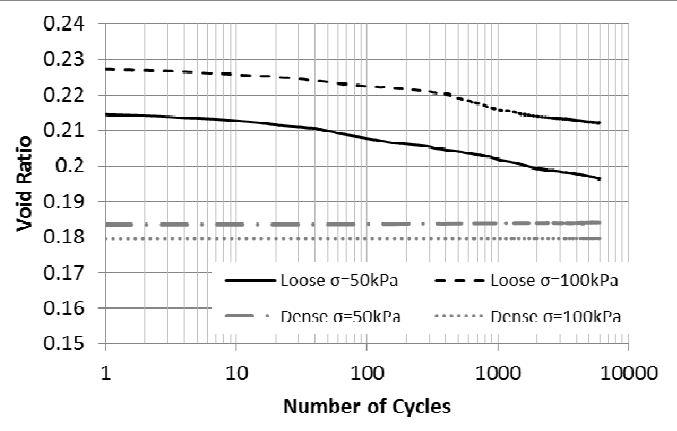

Fig. 6. Evolution of void ratio in simulations.

\subsection{Particle-scale responses}

\subsubsection{Coordination number $\left(N_{c}\right)$}

The coordination number $\left(\mathrm{N}_{\mathrm{c}}\right)$ is the average number of contacts surrounding each particle. It has a strong relation with the stress level within the sample [9]. The evolution of $\mathrm{N}_{\mathrm{c}}$ under cyclic loading for the four samples are shown in Fig. 7. It is clear that the initial low $\mathrm{N}_{\mathrm{c}}$ corresponds to initial low shear stress (thus low shear modulus). The increase in shear modulus for the two loose samples is related to the increase in $\mathrm{N}_{\mathrm{c}}$ and the decrease in shear modulus for the dense sample at $\sigma=50 \mathrm{kPa}$ agrees with the reduce in $\mathrm{N}_{\mathrm{c}}$. However, the evolution $\mathrm{N}_{\mathrm{c}}$ for the dense sample at $\sigma=100 \mathrm{kPa}$ does not follow this trend. There must be other factor dominating the increase of shear modulus for the fourth sample.

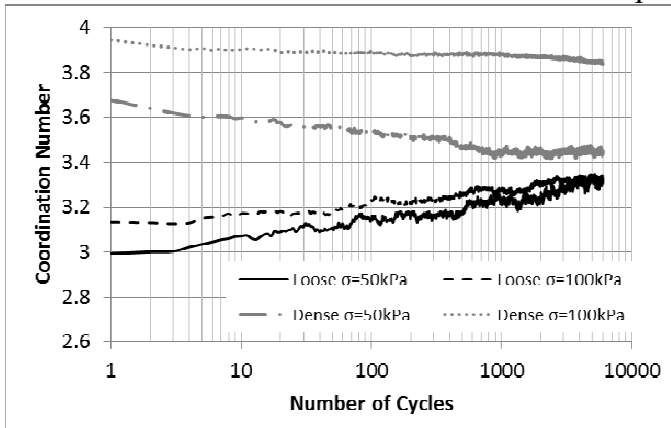

Fig. 7. Evolution of Coordination Number $\left(\mathrm{N}_{\mathrm{c}}\right)$.

\subsubsection{Fabric}

There are many evidences of the impact of fabric anisotropy on characteristics of granular materials, e.g. $[10,11]$. It is worth to analyse the evolution of soil fabric in the current cyclic loading conditions. The spatial distribution of directions of particle contact normals can be quantified using the Fourier approximation [12] as:

$$
E(\theta)=\frac{1}{2 \pi}\left[1+a \cos 2\left(\theta-\theta_{a}\right)\right]
$$

where $a$ is a parameter defining the magnitude of anisotropy and $\theta_{a}$ defines the direction of the principal fabric.

The histogram of spatial distribution of directions of particle contact normals for the dense sample at $\sigma=100 \mathrm{kPa}$ is illustrated in Fig. 8. The Fourier approximation function is indicated by the red ellipse. The major principle fabric of the initial sample is oriented around the horizontal $\left(\theta_{a}=15.0^{\circ}\right)$ as a result of $\mathrm{K}_{0}$ consolidation. When sheared to the maximum strain, the major principle fabric direction rotated to the diagonal direction $\left(\theta_{a} \approx 130.0^{\circ}\right)$; when sheared back to zero strain, the major principle fabric direction rotated to the opposite diagonal direction $\left(\theta_{a} \approx 40.0^{\circ}\right)$. These large rotations of principle fabric in both dense samples only changed in the first loading cycle and remained the same in the remaining cycles. However, the rotation of principle fabric direction occurred slowly in both loose samples. The major principle fabric direction at zero strain rotated slowly from its original direction to $\theta_{a} \approx 40.0^{\circ}$.

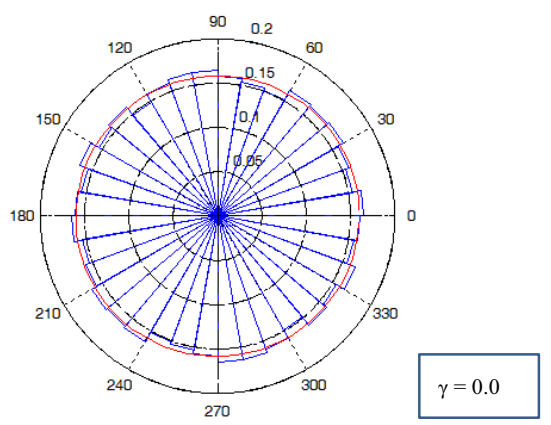

(a) Start of 1st cycle

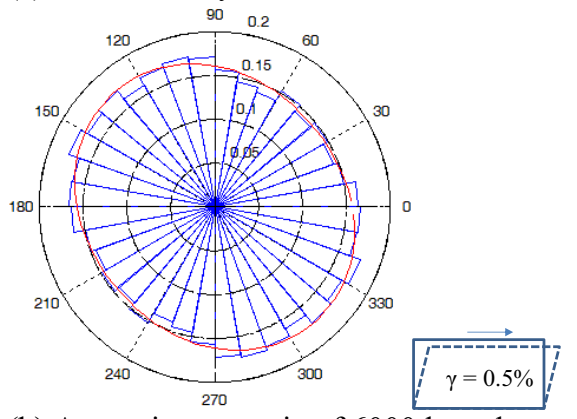

(b) At maximum strain of 6000th cycle 


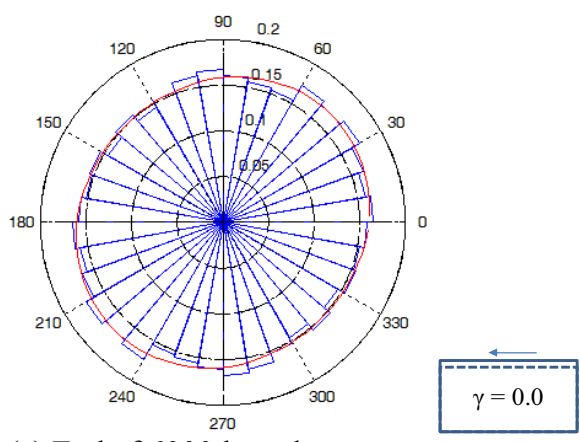

(c) End of 6000th cycle

Fig. 8. Spatial distribution of contact normal.

The difference in the principle fabric directions between the maximum strain and zero strain are plotted in Fig. 9. It is clearly shown that the rotation of principle fabric directions evolved slowly in loose samples but only changed in the first cycle in dense samples. It may be the key reason for the significant volumetric contraction and shear modulus increase in loose samples.

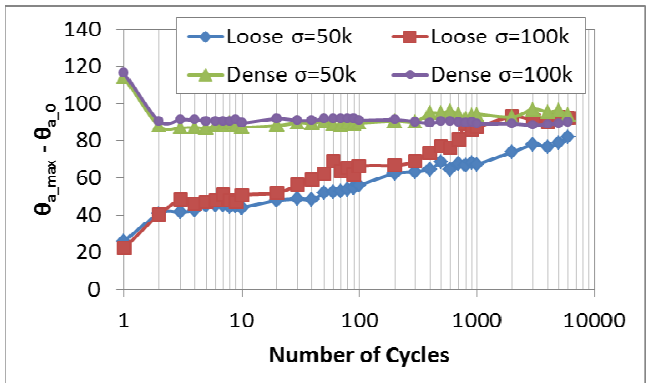

Fig. 9. Difference of major fabric direction between maximum strain and zero strain.

The magnitude of fabric anisotropy is quantified by $a$ in Eq (1). It has been demonstrated previously [10, 11] that larger magnitude of anisotropy can result in higher shear stress. The current four simulations also confirmed this trend. Therefore, the shear modulus, which is determined by $\tau_{\max }-\tau_{0}$, is underlain by magnitudes of anisotropy at corresponding strain values, i.e. $a_{\max }-a_{0}$. The evolutions of $a_{\max }-a_{0}$ for the four simulations are shown in Fig. 10. It is clear that $a_{\max }-a_{0}$ for the dense sample with $\sigma=50 \mathrm{kPa}$ drop significantly to negative values, which agrees with the fact that the $\tau_{\max }-\tau_{0}$ for this sample also drop moderately.

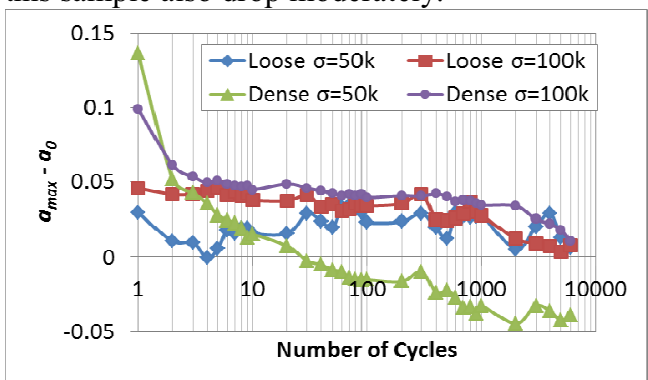

Fig. 10. Difference of magnitude of fabric anisotropy between maximum strain and zero strain.

\section{Discussions and Conclusions}

Both experimental tests and DEM simulations of cyclic simple shear tests were performed to explore the variations of soil characteristics under cyclic loading and its underlying micromechanism. It has been found that:

- Shear modulus for loose soil increases rapidly in the initial loading cycles as a result of soil densification, and then the rate of increase diminishes when void ratio approaches constant;

- The shear modulus increases with increasing vertical stress and relative density, but decreases with increasing strain amplitude as expected.

- Vertical accumulated strain is proportional to the shear strain amplitude but inversely proportional to the relative density of soil;

- Initial relative density has more significant impact on the volumetric response than vertical stress does;

- Higher shear stress level and shear modulus is correlated to higher coordination number;

- The shear stress level and shear modulus is directly correlated to magnitude of fabric anisotropy.

More parametric studies will be carried out in the future work, including two-way cyclic loadings with various ratio of maximum strain to minimum strain. Non-circular disks will be considered as well. An empirical model for evolution of shear modulus will be established to include the impact of all macroscopic and microscopic parameters.

\section{References}

1. S. Bhattacharya, S. Adhikari, Soil Dyn. Earthq. Eng. 31, 805 (2011).

2. S. Bhattacharya, J.A. Cox, D. Lombardi, D. Muir Wood, P. I. Civil Eng.-Geotec., 166, 159 (2012).

3. D. Lombardi, S. Bhattacharya, D. Muir Wood, Soil Dyn. Earthq. Eng. 49, 805 (2013).

4. M. Vucetica, A. Mortezaie, Soil Dyn. Earthq. Eng. 70, 60 (2015)

5. H. Liu, D. Zou, J. Liu, Int. J. Numer. Anal. Met, 38, 1503 (2014).

6. ASTM D6528 - 07, (2007).

7. P. Cuéllar, S. Georgi, M. Baeßler, W. Rücker., Granul. Matter, 14, 11 (2012).

8. Itasca, PFC2D manual 4.0 (2008).

9. L. Cui, C. O’Sullivan, S. O’Neill, Geotechnique, 57, 831 (2007)

10. X. Li, H.S. Yu, Acta Mech., 225, 2345 (2014).

11. L. Cui, C. O’Sullivan, Geotechnique, 56, 455 (2006).

12. L. Rothenburg, R.J. Bathurst, Geotechnique, 39, 601, (1989). 